\title{
Existence theorems for a semilinear elliptic boundary value problem
}

\author{
by Salvatore A. Marano (Catania)
}

Abstract. Let $\Omega$ be a bounded domain in $\mathbb{R}^{n}, n \geq 3$, with a smooth boundary $\partial \Omega$; let $L$ be a linear, second order, elliptic operator; let $f$ and $g$ be two real-valued functions defined on $\Omega \times \mathbb{R}$ such that $f(x, z) \leq g(x, z)$ for almost every $x \in \Omega$ and every $z \in \mathbb{R}$. In this paper we prove that, under suitable assumptions, the problem

$$
\begin{cases}f(x, u) \leq L u \leq g(x, u) & \text { in } \Omega, \\ u=0 & \text { on } \partial \Omega,\end{cases}
$$

has at least one strong solution $u \in W^{2, p}(\Omega) \cap W_{0}^{1, p}(\Omega)$. Next, we present some remarkable special cases.

Introduction. Let $\Omega$ be a bounded domain in $\mathbb{R}^{n}, n \geq 3$, with a smooth boundary $\partial \Omega$; let $L$ be a linear, second order, elliptic differential operator; let $f$ and $g$ be two real-valued functions defined on $\Omega \times \mathbb{R}$ such that $f(x, z) \leq$ $g(x, z)$ for almost every $x \in \Omega$ and every $z \in \mathbb{R}$.

Consider the problem

$$
\begin{cases}f(x, u) \leq L u \leq g(x, u) & \text { in } \Omega, \\ u=0 & \text { on } \partial \Omega .\end{cases}
$$

A function $u: \Omega \rightarrow \mathbb{R}$ is said to be a strong solution of $(\mathrm{P})$ if $u \in W^{2, p}(\Omega) \cap$ $\left.W_{0}^{1, p}(\Omega), p \in\right] n / 2, \infty[$, and, for almost every $x \in \Omega$, one has $f(x, u(x)) \leq$ $L u(x) \leq g(x, u(x))$.

Remarkable special cases of problem $(\mathrm{P})$ are those where $f(x, z)=$ $g(x, z),(x, z) \in \Omega \times \mathbb{R}$, or, roughly speaking, $f(x, z)=\liminf _{w \rightarrow z} \varphi(x, w)$ and $g(x, z)=\lim \sup _{w \rightarrow z} \varphi(x, w),(x, z) \in \Omega \times \mathbb{R}$, with $\varphi$ a locally bounded real-valued function defined on $\Omega \times \mathbb{R}$. Both have been extensively studied, mainly by variational methods $[6],[11],[15]$, or topological methods [5], [17], or sub- and super-solution arguments [9], [14].

1991 Mathematics Subject Classification: 35R70, 35J60.

Key words and phrases: elliptic differential inclusions, semilinear elliptic equations, strong solutions. 
Our approach is quite different and follows that introduced in [10] with regard to the Dirichlet problem for ordinary differential inclusions. In this way, we obtain an existence result (Theorem 2.1) for strong solutions to problem (P) where rather general conditions on the functions $f$ and $g$ are assumed. For instance, we do not need that the functions $x \rightarrow f(x, z)$, $x \rightarrow g(x, z), x \in \Omega$, are measurable for all $z \in \mathbb{R}$, but only for $z$ in a dense subset of $\mathbb{R}$. Afterwards, we emphasize two special cases of Theorem 2.1 (Theorems 2.2 and 2.3). Theorem 2.2 has an overlap with Theorem 5.1 of [6]. As a simple consequence of Theorem 2.3, we obtain a result (Theorem 2.4) which improves, in several concrete cases, Theorem 3.3 of [17], dealing with an elliptic problem with critical Sobolev exponent.

The main tools we use to establish our results are Theorem 1 of [13] and Theorem 3.1 of [2].

1. Preliminaries. Let $X$ and $Y$ be two nonempty sets. A multifunction $\Phi$ from $X$ into $Y$ (briefly, $\Phi: X \rightarrow 2^{Y}$ ) is a function from $X$ into the family of all subsets of $Y$. The graph of $\Phi$ is the set $\{(x, y) \in X \times Y: y \in \Phi(x)\}$. If $W \subseteq Y$, we set $\Phi^{-}(W)=\{x \in X: \Phi(x) \cap W \neq \emptyset\}$. If $(X, \mathcal{F})$ is a measurable space and $Y$ is a topological space, we say that $\Phi$ is measurable if $\Phi^{-}(W) \in \mathcal{F}$ for every open set $W \subseteq Y$. If $X$ and $Y$ are two topological spaces, we say that $\Phi$ is upper semicontinuous if, for every closed set $W \subseteq Y$, the set $\Phi^{-}(W)$ is closed in $X$. If $(X, d)$ is a metric space, for every $x \in X$ and every nonempty set $W \subseteq X$, we define $d(x, W)=\inf _{z \in W} d(x, z)$.

In the sequel we shall apply the following proposition, whose simple proof follows immediately from Theorem 3.5 of [8].

Proposition 1.1. Let $(X, \mathcal{F})$ be a measurable space and let $\varphi$ be a measurable real-valued function defined on $X$. Then the multifunctions $x \rightarrow$ ]$-\infty, \varphi(x)]$ and $x \rightarrow[\varphi(x), \infty[, x \in X$, are measurable.

We shall also use the following proposition, which can be easily verified.

Proposition 1.2. Let $X$ be a topological space and let $\varphi$ be an upper (resp. lower) semicontinuous real-valued function defined on $X$. Then the multifunction $x \rightarrow]-\infty, \varphi(x)]$ (resp. $x \rightarrow[\varphi(x), \infty[), x \in X$, is upper semicontinuous.

Let $n$ be a positive integer and let $\mathbb{R}^{n}$ be the real Euclidean $n$-space. A nonempty set $\Omega \subseteq \mathbb{R}^{n}$ is said to be a domain if it is open and connected. By $\bar{\Omega}$ (resp. $\partial \Omega$ ) we denote the closure (resp. the boundary) of $\Omega$ in $\mathbb{R}^{n}$.

As regards the function spaces we shall use in the sequel, our notations are standard; we refer for instance to [1], [7].

For the reader's convenience, we report now the statement of Theorem 1 in [13], which will be applied in the sequel. 
TheOREM 1.1. Let $(T, \mathcal{F}, \mu)$ be a finite, nonatomic, complete measure space; let $V$ be a nonempty set; let $\left(X,\|\cdot\|_{X}\right)$ and $\left(Y,\|\cdot\|_{Y}\right)$ be two separable real Banach spaces, with $Y$ finite-dimensional; let $p, q, s \in[1, \infty]$, with $q<$ $\infty$ and $q \leq p \leq s$; let $\Psi: V \rightarrow L^{s}(T, Y)$ be a surjective and one-to-one operator; let $\Phi: V \rightarrow L^{1}(T, X)$ be an operator such that, for every $v \in$ $L^{s}(T, Y)$ and every sequence $\left\{v_{n}\right\}$ in $L^{s}(T, Y)$, weakly converging to $v$ in $L^{q}(T, Y)$, the sequence $\left\{\Phi\left(\Psi^{-1}\left(v_{n}\right)\right)\right\}$ converges strongly to $\Phi\left(\Psi^{-1}(v)\right)$ in $L^{1}(T, X)$; let $\varphi:[0, \infty[\rightarrow[0, \infty]$ be a nondecreasing function such that

$$
\underset{t \in T}{\operatorname{ess} \sup }\|\Phi(u)(t)\|_{X} \leq \varphi\left(\|\Psi(u)\|_{L^{p}(T, Y)}\right)
$$

for all $u \in V$. Further, let $F: T \times X \rightarrow 2^{Y}$ be a multifunction, with nonempty, convex, closed values, satisfying the following conditions:

(i) For $\mu$-almost every $t \in T$, the multifunction $F(t, \cdot)$ has closed graph.

(ii) The set $\{x \in X: F(\cdot, x)$ is $\mathcal{F}$-measurable $\}$ is dense in $X$.

(iii) There exists a real number $r>0$ such that the function

$$
t \rightarrow \sup _{\|x\|_{X} \leq \varphi(r)} d\left(0_{Y}, F(t, x)\right)
$$

belongs to $L^{s}(T)$ and its norm in $L^{p}(T)$ is less than or equal to $r$ ( $d$ is the metric induced by $\|\cdot\|_{Y}$ and $0_{Y}$ denotes the zero vector of $Y$ ).

Under these assumptions, there exists $\widetilde{u} \in V$ such that

$$
\Psi(\widetilde{u})(t) \in F(t, \Phi(\widetilde{u})(t)) \quad \text { and } \quad\|\Psi(\widetilde{u})(t)\|_{Y} \leq \sup _{\|x\|_{X} \leq \varphi(r)} d\left(0_{Y}, F(t, x)\right)
$$

$\mu$-almost everywhere in $T$.

2. Results. Let $\Omega$ be a bounded domain in $\mathbb{R}^{n}, n \geq 3$, with a $C^{1,1}$ boundary, and let $L$ be the linear elliptic operator

$$
L u=-\sum_{i, j=1}^{n} a_{i j}(x) \frac{\partial^{2} u}{\partial x_{i} \partial x_{j}}+\sum_{i=1}^{n} b_{i}(x) \frac{\partial u}{\partial x_{i}}+c(x) u
$$

where: $a_{i j} \in C^{1}(\bar{\Omega}), a_{i j}=a_{j i}$ for every $i, j=1, \ldots, n$, and $\sum_{i, j=1}^{n} a_{i j}(x) \xi_{i} \xi_{j}$ $\geq \xi_{1}^{2}+\ldots+\xi_{n}^{2}$ for all $x \in \Omega,\left(\xi_{1}, \ldots, \xi_{n}\right) \in \mathbb{R}^{n} ; b_{i} \in L^{\infty}(\Omega)$ for every $i=1, \ldots, n ; c \in L^{\infty}(\Omega)$ and $c(x) \geq 0$ for almost all $x \in \Omega$.

It is well known that, for any $p \in] 1, \infty[, L$ is a one-to-one operator from $W^{2, p}(\Omega) \cap W_{0}^{1, p}(\Omega)$ onto $L^{p}(\Omega)$ (see, for instance, [7, Theorem 9.15]).

Denote by $\omega_{n}$ the volume of the unit ball in $\mathbb{R}^{n}$ and set

$$
\beta=\operatorname{esssup}_{x \in \Omega}\left[\sum_{i=1}^{n}\left(b_{i}(x)+\sum_{j=1}^{n} \frac{\partial a_{i j}(x)}{\partial x_{j}}\right)^{2}\right]^{1 / 2} .
$$


When $p>n / 2$, owing to Theorem 3.1 of [2], for every $u \in W^{2, p}(\Omega) \cap W_{0}^{1, p}(\Omega)$ one has

$$
\underset{x \in \Omega}{\operatorname{ess} \sup }|u(x)| \leq B\|L u\|_{L^{p}(\Omega)},
$$

where

(1) $B=\frac{1}{n^{2} \omega_{n}^{2 / n}}$

$$
\times\left[\int_{0}^{m(\Omega)}\left(e^{-\beta\left(r / \omega_{n}\right)^{1 / n}} \int_{r}^{m(\Omega)} s^{-2+2 / n} e^{\beta\left(s / \omega_{n}\right)^{1 / n}} d s\right)^{p /(p-1)} d r\right]^{1-1 / p} ;
$$

if $\beta=0$, then a simple computation shows that the constant $B$ becomes $[16$, Theorem 2]

$$
\begin{aligned}
B=[ & m(\Omega)]^{2 / n-1 / p} \frac{\Gamma(1+n / 2)^{2 / n}}{n(n-2) \pi} \\
& \times\left[\frac{\Gamma(1+p /(p-1)) \Gamma(n /(n-2)-p /(p-1))}{\Gamma(n /(n-2))}\right]^{1-1 / p}
\end{aligned}
$$

$\left(\|\cdot\|_{L^{p}(\Omega)}\right.$ denotes the usual norm of $L^{p}(\Omega), m(\Omega)$ is the Lebesgue measure of $\Omega$ and $\Gamma$ is the Gamma function).

The main result of this paper is the following

THEOREM 2.1. Let $f$ and $g$ be two real-valued functions defined on $\Omega \times \mathbb{R}$. Assume that:

$\left(\mathrm{i}_{1}\right)$ For almost every $x \in \Omega$ and every $z \in \mathbb{R}$, one has $f(x, z) \leq g(x, z)$.

$\left(\mathrm{i}_{2}\right)$ For almost every $x \in \Omega$ the function $z \rightarrow f(x, z)$ is lower semicontinuous and the function $z \rightarrow g(x, z)$ is upper semicontinuous.

$\left(\mathrm{i}_{3}\right)$ There exists a set $D \subseteq \mathbb{R}$, with $\bar{D}=\mathbb{R}$, such that, for each $z \in D$, the functions $x \rightarrow f(x, z)$ and $x \rightarrow g(x, z)$ are measurable.

$\left(\mathrm{i}_{4}\right)$ There exist $\left.p \in\right] n / 2, \infty[$ and $r>0$ such that the function

$$
x \rightarrow \sup _{|z| \leq B r} \max \{-g(x, z), \max \{0, f(x, z)\}\},
$$

where $B$ is given by $(1)$, belongs to $L^{p}(\Omega)$ and its norm in $L^{p}(\Omega)$ is less than or equal to $r$.

Then problem $(\mathrm{P})$ has at least one strong solution $u \in W^{2, p}(\Omega) \cap$ $W_{0}^{1, p}(\Omega)$. Moreover, for almost every $x \in \Omega$, one has

$$
|L u(x)| \leq \sup _{|z| \leq B r} \max \{-g(x, z), \max \{0, f(x, z)\}\} .
$$

Proof. Let us apply Theorem 1.1. To this end, choose: $T=\Omega$ with the Lebesgue measure structure $\left(\mathcal{L}_{\Omega}, m\right) ; V=W^{2, p}(\Omega) \cap W_{0}^{1, p}(\Omega) ; X=Y=\mathbb{R}$; $q=s=p ; \Psi(u)=L u$ for all $u \in V ; \Phi(u)=u$ for all $u \in V ; \varphi(\lambda)=B \lambda$ for 
all $\lambda \in[0, \infty$, where $B$ is given by (1). From Theorem 9.15 of [7] it follows that $\Psi$ is a one-to-one operator from $V$ onto $L^{p}(\Omega)$. Let $v \in L^{p}(\Omega)$ and let $\left\{v_{k}\right\}$ be a sequence in $L^{p}(\Omega)$ weakly converging to $v$ in $L^{p}(\Omega)$. Bearing in mind that $\Psi^{-1}$ is a continuous, linear operator from $L^{p}(\Omega)$ into $W^{2, p}(\Omega)$ (see, for instance, [7, Lemma 9.17]), we deduce that $\left\{\Psi^{-1}\left(v_{k}\right)\right\}$ converges weakly to $\Psi^{-1}(v)$ in $W^{2, p}(\Omega)$. Therefore, by the Rellich-Kondrachov Theorem [1, Theorem 6.2$]$, it also converges to $\Psi^{-1}(v)$ in $C^{0}(\bar{\Omega})$. This implies $\lim _{k \rightarrow \infty} \Phi\left(\Psi^{-1}\left(v_{k}\right)\right)=\Phi\left(\Psi^{-1}(v)\right)$ in $L^{1}(\Omega)$.

Next, observe that, owing to Theorem 3.1 of [2], for every $u \in V$ one has

$$
\operatorname{ess}_{x \in \Omega}|\Phi(u)(x)| \leq \varphi\left(\|\Psi(u)\|_{L^{p}(\Omega)}\right)
$$

Now, let $\Omega_{1} \in \mathcal{L}_{\Omega}$ be such that $m\left(\Omega_{1}\right)=0$ and $\left(\mathrm{i}_{1}\right),\left(\mathrm{i}_{2}\right)$ hold for all $x \in \Omega \backslash \Omega_{1}$. For every $(x, z) \in \Omega \times \mathbb{R}$, we define

$$
F(x, z)=\mathbb{R} \quad \text { if } x \in \Omega_{1}, \quad F(x, z)=[f(x, z), g(x, z)] \quad \text { if } x \in \Omega \backslash \Omega_{1} .
$$

Obviously, the multifunction $F: \Omega \times \mathbb{R} \rightarrow 2^{\mathbb{R}}$ so defined is nonempty, convex, closed-valued. Let us prove that $F$ satisfies the assumptions (i)-(iii) of Theorem 1.1. To this end, fix $x \in \Omega \backslash \Omega_{1}$. By ( $\mathrm{i}_{2}$ ) and Proposition 1.2, the multifunctions $z \rightarrow[f(x, z), \infty[$ and $z \rightarrow]-\infty, g(x, z)], z \in \mathbb{R}$, are upper semicontinuous. So, by Theorems 1.3.2 and 1.2.12 in [4], the multifunction $z \rightarrow[f(x, z), g(x, z)]$ has closed graph. Next, fix $z \in D$. Owing to $\left(\mathrm{i}_{3}\right)$ and Proposition 1.1, the multifunctions $x \rightarrow]-\infty, g(x, z)]$ and $x \rightarrow[f(x, z), \infty[$, $x \in \Omega \backslash \Omega_{1}$, are measurable. Hence, by [8, Corollary 4.2], the multifunction $x \rightarrow[f(x, z), g(x, z)], x \in \Omega \backslash \Omega_{1}$, is measurable. This implies that the multifunction $x \rightarrow F(x, z), x \in \Omega$, is measurable.

Finally, observe that, for every $x \in \Omega \backslash \Omega_{1}$ and every $z \in \mathbb{R}$, one has

$$
d(0, F(x, z))=\max \{-g(x, z), \max \{0, f(x, z)\}\} .
$$

Therefore, by $\left(\mathrm{i}_{4}\right)$, the function $x \rightarrow \sup _{|z| \leq \varphi(r)} d(0, F(x, z))$ belongs to $L^{p}(\Omega)$ and its norm in this space is less than or equal to $r$.

At this point we can apply Theorem 1.1. Thus, there exists $u \in W^{2, p}(\Omega)$ $\cap W_{0}^{1, p}(\Omega)$ such that $L u(x) \in F(x, u(x))$ and $|L u(x)| \leq \sup _{|z| \leq B r} d(0, F(x, z))$ for almost every $x \in \Omega$. This completes the proof.

R e m a r k 2.1. The assumptions $\left(\mathrm{i}_{2}\right)$ and $\left(\mathrm{i}_{3}\right)$ of Theorem 2.1 do not imply that the functions $x \rightarrow f(x, z)$ and $x \rightarrow g(x, z), x \in \Omega$, are measurable for all $z \in \mathbb{R}$. In fact, let $\Omega_{0}$ be a nonmeasurable subset of $\Omega$, let $D$ be the set of all rational numbers and let $H$ be a closed subset of $\mathbb{R} \backslash D$. For every $(x, z) \in \Omega \times \mathbb{R}$, we set

$$
f(x, z)=\left\{\begin{array}{ll}
-1 & \text { if }(x, z) \in \Omega_{0} \times H, \\
0 & \text { if }(x, z) \in(\Omega \times \mathbb{R}) \backslash\left(\Omega_{0} \times H\right),
\end{array} \quad g(x, z)=-f(x, z) .\right.
$$


An easy computation shows that $f, g$ satisfy all the assumptions of Theorem 2.1. Nevertheless, for every $z \in H$, the functions $x \rightarrow f(x, z)$ and $x \rightarrow g(x, z), x \in \Omega$, are not measurable.

The next result is concerned with an interesting special case of problem (P). In proving it, we shall use the following lemmas.

LEMMA 2.1. Let $\varphi$ be a real-valued function defined on $\Omega \times \mathbb{R}$. Suppose that the function $\varphi(x, \cdot)$ is measurable for all $x \in \Omega$ and one has $\sup _{(x, z) \in \Omega \times]-\varrho, \varrho[}|\varphi(x, z)|<\infty$ for all $\varrho>0$. For every $(x, z) \in \Omega \times \mathbb{R}$, we define

$$
\underline{\varphi}(x, z)=\lim _{\sigma \rightarrow 0^{+}} \operatorname{essinf}_{|w-z|<\sigma} \varphi(x, w), \quad \bar{\varphi}(x, z)=\lim _{\sigma \rightarrow 0^{+}} \operatorname{ess} \sup \varphi(x, w|<\sigma|
$$

Then, for any $x \in \Omega$, the function $z \rightarrow \varphi(x, z)$ is lower semicontinuous and the function $z \rightarrow \bar{\varphi}(x, z)$ is upper semicontinuous.

The proof of the preceding lemma is straightforward; so we omit it.

LEMma 2.2. Let $\Omega$ be with a $C^{2, \alpha}$-boundary for some $\left.\alpha \in\right] 0,1\left[; \Omega_{0} \subseteq \Omega\right.$ a domain; $\lambda_{1}$ the first eigenvalue of $-\Delta=-\sum_{i=1}^{n} \partial^{2} / \partial x_{i}^{2}$ with the homogeneous Dirichlet boundary condition; $\lambda \in]-\infty, \lambda_{1}[$. Moreover, let $v \in$ $W^{2, p}\left(\Omega_{0}\right), p>n$, be such that

$$
\begin{aligned}
& -\Delta v(x)-\lambda v(x) \leq 0 \quad \text { for almost every } x \in \Omega_{0}, \\
& v(x) \leq 0 \quad \text { for every } x \in \partial \Omega_{0} .
\end{aligned}
$$

Then $v(x) \leq 0$ for every $x \in \Omega_{0}$.

Proof. Owing to Theorem 1.17 of [3], there is $v_{0} \in C^{2}(\Omega) \cap C^{1}(\bar{\Omega})$ such that $-\Delta v_{0}(x)-\lambda v_{0}(x)=1, v_{0}(x)>0$ for all $x \in \Omega$, and $v_{0}(x)=0$ for all $x \in \partial \Omega$. Moreover, by Theorem 36.VI of [12], $v_{0} \in C^{2}(\bar{\Omega})$. Choose $\sigma>0$ satisfying $1-\lambda \sigma>0$ and set, for every $x \in \bar{\Omega}, w(x)=v_{0}(x)+\sigma$. Then one has

(3) $\quad-\Delta w(x)-\lambda w(x)>0 \quad$ for all $x \in \Omega, \quad w(x)>0 \quad$ for all $x \in \bar{\Omega}$.

Now, define $u(x)=v(x) / w(x), x \in \Omega_{0}$, and observe that $u \in W^{2, p}\left(\Omega_{0}\right)$ and that, by (2), for almost every $x \in \Omega_{0}$, one has

$$
w(x) \Delta u(x)+\sum_{i=1}^{n} \frac{\partial w(x)}{\partial x_{i}} \frac{\partial u(x)}{\partial x_{i}}+(\Delta w(x)+\lambda w(x)) u(x) \geq 0 .
$$

Since Theorems 3.I and 3.V of [12], together with (3), yield $\max _{x \in \bar{\Omega}_{0}} u(x) \leq$ 0 , we obtain $v(x) \leq 0$ for every $x \in \Omega_{0}$.

We are now in a position to establish the following

TheOREM 2.2. Let $\Omega$ be with a $C^{2, \alpha}$-boundary for some $\left.\alpha \in\right] 0,1[$ and let $\varphi$ be a real-valued function defined on $\Omega \times \mathbb{R}$. Suppose that: 
$\left(\mathrm{j}_{1}\right)$ The function $z \rightarrow \varphi(x, z)$ is measurable for all $x \in \Omega$. $\leq k_{\varrho}$.

$\left(\mathrm{j}_{2}\right)$ For every $\varrho>0$ there is $k_{\varrho}>0$ such that $\sup _{(x, z) \in \Omega \times]-\varrho, \varrho[}|\varphi(x, z)|$

$\left(\mathrm{j}_{3}\right)$ The functions $x \rightarrow \underline{\varphi}(x, z)$ and $x \rightarrow \bar{\varphi}(x, z)$ are measurable for all $z \in \mathbb{R}(\underline{\varphi}$ and $\bar{\varphi}$ are as in Lemma 2.1$)$.

$\left(\mathrm{j}_{4}\right) \limsup _{z \rightarrow \pm \infty} \varphi(x, z) / z<\lambda_{1}$ uniformly with respect to $x \in \Omega$, where $\lambda_{1}$ is as in Lemma 2.2 .

Then the problem

$$
\begin{cases}\underline{\varphi}(x, u) \leq-\Delta u \leq \bar{\varphi}(x, u) & \text { in } \Omega \\ u=0 & \text { on } \partial \Omega\end{cases}
$$

has at least one solution $u \in W^{2, p}(\Omega) \cap W_{0}^{1, p}(\Omega), p>n$.

Proof. It is well known that $\lambda_{1}>0$. Hence, by $\left(\mathrm{j}_{4}\right)$, there are $\left.\varepsilon \in\right] 0, \lambda_{1}[$ and $M>0$ such that

$$
\begin{array}{ll}
\varphi(x, z)<\left(\lambda_{1}-\varepsilon\right) z+M & \text { for every }(x, z) \in \Omega \times[0, \infty[, \\
\varphi(x, z)>\left(\lambda_{1}-\varepsilon\right) z-M & \text { for every }(x, z) \in \Omega \times]-\infty, 0] .
\end{array}
$$

Owing to [3, Theorem 1.17], there exists $u_{0} \in C^{2}(\Omega) \cap C^{1}(\bar{\Omega})$ satisfying

$$
\begin{aligned}
-\Delta u_{0}(x) & =\left(\lambda_{1}-\varepsilon\right) u_{0}(x)+M, \quad u_{0}(x)>0 \quad \text { for all } x \in \Omega, \\
u_{0}(x) & =0 \quad \text { for all } x \in \partial \Omega .
\end{aligned}
$$

Now, let $\varrho=\max _{x \in \bar{\Omega}}\left|u_{0}(x)\right|$. For every $(x, z) \in \Omega \times \mathbb{R}$, we set

$$
f(x, z)=\left\{\begin{array}{ll}
\underline{\varphi}(x, \varrho) & \text { if } z \geq \varrho, \\
\underline{\varphi}(x, z) & \text { if }|z|<\varrho, \\
\underline{\varphi}(x,-\varrho) & \text { if } z \leq-\varrho,
\end{array} \quad g(x, z)= \begin{cases}\bar{\varphi}(x, \varrho) & \text { if } z \geq \varrho, \\
\bar{\varphi}(x, z) & \text { if }|z|<\varrho, \\
\bar{\varphi}(x,-\varrho) & \text { if } z \leq-\varrho .\end{cases}\right.
$$

Obviously, the functions $f, g: \Omega \times \mathbb{R} \rightarrow \mathbb{R}$ so defined satisfy the assumption $\left(\mathrm{i}_{1}\right)$ of Theorem 2.1. Moreover, by (4), one has

$$
\begin{array}{ll}
g(x, z) \leq\left(\lambda_{1}-\varepsilon\right) z+M & \text { for every }(x, z) \in \Omega \times] 0, \infty[, \\
f(x, z) \geq\left(\lambda_{1}-\varepsilon\right) z-M & \text { for every }(x, z) \in \Omega \times]-\infty, 0[.
\end{array}
$$

Bearing in mind Lemma 2.1, it is a simple matter to see that $f, g$ satisfy $\left(\mathrm{i}_{2}\right)$ of Theorem 2.1. Finally, since $\left(\mathrm{i}_{3}\right)$ of Theorem 2.1 , with $D=\mathbb{R}$, follows immediately from $\left(\mathrm{j}_{3}\right)$, to apply this result it is sufficient to show that $\left(\mathrm{i}_{4}\right)$ holds. Observe that, by $\left(\mathrm{j}_{2}\right)$, one has

$$
\begin{aligned}
& \sup _{(x, z) \in \Omega \times \mathbb{R}}|f(x, z)| \leq \sup _{(x, z) \in \Omega \times]-\varrho-1, \varrho+1[}|\underline{\varphi}(x, z)| \leq k_{\varrho+1}, \\
& \sup _{(x, z) \in \Omega \times \mathbb{R}}|g(x, z)| \leq \sup _{(x, z) \in \Omega \times]-\varrho-1, \varrho+1[}|\bar{\varphi}(x, z)| \leq k_{\varrho+1} .
\end{aligned}
$$


So, if we choose $p>n$ and $r>k_{\varrho+1}[m(\Omega)]^{1 / p}$, we have

$$
\begin{aligned}
\left(\int_{\Omega}\left(\sup _{|z| \leq B r} \max \{-g(x, z), \max \{0, f(x, z)\}\}\right)^{p} d x\right)^{1 / p} & \\
& \leq k_{\varrho+1}[m(\Omega)]^{1 / p}<r .
\end{aligned}
$$

By Theorem 2.1, there exists $u \in W^{2, p}(\Omega) \cap W_{0}^{1, p}(\Omega)$ satisfying

$$
f(x, u(x)) \leq-\Delta u(x) \leq g(x, u(x))
$$

almost everywhere in $\Omega$. Let us prove that $u(x) \leq u_{0}(x)$ for every $x \in \Omega$. To this end, set $\Omega_{1}=\{x \in \Omega: u(x)>0\}$. If $\Omega_{1}=\emptyset$ our claim is obvious; otherwise, denoting by $\Omega_{1}^{*}$ a connected component of $\Omega_{1}$, owing to (5)-(7), we have

$$
\begin{aligned}
-\Delta\left(u(x)-u_{0}(x)\right) & \leq g(x, u(x))-\left(\lambda_{1}-\varepsilon\right) u_{0}(x)-M \\
& \leq\left(\lambda_{1}-\varepsilon\right) u(x)+M-\left(\lambda_{1}-\varepsilon\right) u_{0}(x)-M \\
& =\left(\lambda_{1}-\varepsilon\right)\left(u(x)-u_{0}(x)\right)
\end{aligned}
$$

almost everywhere in $\Omega_{1}^{*}$, and $u(x)-u_{0}(x) \leq 0$ for every $x \in \partial \Omega_{1}^{*}$. By Lemma 2.2, this implies $u(x)-u_{0}(x) \leq 0$ for all $x \in \Omega_{1}^{*}$. Hence, $u(x) \leq u_{0}(x)$ for every $x \in \Omega$. In a similar way it is possible to verify that $u(x) \geq-u_{0}(x)$ for all $x \in \Omega$.

Thus, the conclusion follows immediately from (7) and the fact that, for every $x \in \Omega$, one has $|u(x)| \leq \varrho$.

Remark 2.2. We observe that, if the function $\varphi$ does not depend on $x$ and $\lim _{w \rightarrow z^{-}} \varphi(w)$ and $\lim _{w \rightarrow z^{+}} \varphi(w)$ exist for each $z \in \mathbb{R}$, then Theorem 2.2 and Theorem 5.1 of [6] coincide (see [6], Example 1 and Theorem 5.1).

If $f(x, z)=g(x, z),(x, z) \in \Omega \times \mathbb{R}$, Theorem 2.1 assumes the following form.

THEOREM 2.3. Let $f$ be a real-valued function defined on $\Omega \times \mathbb{R}$. Assume that:

$\left(\mathrm{k}_{1}\right)$ For almost every $x \in \Omega$, the function $z \rightarrow f(x, z)$ is continuous.

$\left(\mathrm{k}_{2}\right)$ For every $z \in \mathbb{R}$, the function $x \rightarrow f(x, z)$ is measurable.

$\left(\mathrm{k}_{3}\right)$ There exist $\left.p \in\right] n / 2, \infty[$ and $r>0$ such that the function

$$
x \rightarrow \sup _{|z| \leq B r}|f(x, z)|,
$$

where $B$ is given by $(1)$, belongs to $L^{p}(\Omega)$ and its norm in this space is less than or equal to $r$.

Then the problem

$$
\begin{cases}L u=f(x, u) & \text { in } \Omega \\ u=0 & \text { on } \partial \Omega\end{cases}
$$


has at least one strong solution $u \in W^{2, p}(\Omega) \cap W_{0}^{1, p}(\Omega)$. Moreover, for almost every $x \in \Omega$, one has $|L u(x)| \leq \sup _{|z| \leq B r}|f(x, z)|$.

Remark 2.3. A simple sufficient condition in order that $\left(\mathrm{k}_{3}\right)$ of Theorem 2.3 holds is the following.

$\left(\mathrm{k}_{3}^{\prime}\right)$ There exist $\left.p \in\right] n / 2, \infty[, \gamma \in] 0, \infty\left[\right.$ and $\alpha, \beta \in L^{p}(\Omega)$ such that

$$
|f(x, z)| \leq \alpha(x)+\beta(x)|z|^{\gamma}
$$

for almost every $x \in \Omega$ and every $z \in \mathbb{R}$, and, if $\|\beta\|_{L^{p}(\Omega)}>0$, then either

$$
B\|\beta\|_{L^{p}(\Omega)}<1 \quad \text { or } \quad\|\alpha\|_{L^{p}(\Omega)} \leq \frac{\gamma-1}{\gamma}\left(\frac{1}{\gamma B^{\gamma}\|\beta\|_{L^{p}(\Omega)}}\right)^{1 /(\gamma-1)},
$$

according to whether $\gamma=1$ or $\gamma>1$.

We verify this only for $\|\beta\|_{L^{p}(\Omega)}>0$ and $\gamma>1$, since in the other cases the proof is similar. To this end, choose $r=\left[\gamma B^{\gamma}\|\beta\|_{L^{p}(\Omega)}\right]^{-1 /(\gamma-1)}$. Then, by (8), we have

$$
\begin{aligned}
& \left(\int_{\Omega}\left(\sup _{|z| \leq B r}|f(x, z)|\right)^{p} d x\right)^{1 / p} \\
& \leq\|\alpha\|_{L^{p}(\Omega)}+(B r)^{\gamma}\|\beta\|_{L^{p}(\Omega)} \\
& \leq \frac{\gamma-1}{\gamma}\left(\frac{1}{\gamma B^{\gamma}\|\beta\|_{L^{p}(\Omega)}}\right)^{1 /(\gamma-1)}+B^{\gamma}\|\beta\|_{L^{p}(\Omega)}\left(\frac{1}{\gamma B^{\gamma}\|\beta\|_{L^{p}(\Omega)}}\right)^{\gamma /(\gamma-1)} .
\end{aligned}
$$

For other existence results for problem $\left(\mathrm{P}_{1}\right)$ where one assumes that $f$ satisfies a growth condition like (8), we refer for instance to [11], [17].

In particular, we emphasize that Theorem 3.3 of [17] is improved, in several concrete cases (pick, for example, $n=4$ and $p=3$ ), by the following result, which is an immediate consequence of Theorem 2.3.

Theorem 2.4. Let $p \in] n / 2, \infty\left[\right.$ and let $h \in L^{p}(\Omega)$. Suppose that

$$
\|h\|_{L^{p}(\Omega)} \leq[m(\Omega)]^{-(n-2) /(4 p)} B^{-(n+2) / 4} \frac{4}{n+2}\left(\frac{n-2}{n+2}\right)^{(n-2) / 4},
$$

where $B$ is given by $(1)^{\prime}$. Then the problem

$$
\begin{cases}\Delta u+|u|^{(n+2) /(n-2)}+h(x)=0 & \text { in } \Omega \\ u=0 & \text { on } \partial \Omega\end{cases}
$$

has at least one strong solution $u \in W^{2, p}(\Omega) \cap W_{0}^{1, p}(\Omega)$.

Finally, we present a very simple example of an application of Theorem 2.3, where it seems that it is impossible to apply any of the results just cited. 
EXAMPle 2.1. Let $p \in] n / 2, \infty\left[\right.$ and let $h \in L^{p}(\Omega)$ be such that

$$
\|h\|_{L^{p}(\Omega)} \leq \frac{1}{B e} .
$$

Then the problem

$$
\begin{cases}L u=h(x) e^{|u|} & \text { in } \Omega, \\ u=0 & \text { on } \partial \Omega,\end{cases}
$$

has at least one solution $u \in W^{2, p}(\Omega) \cap W_{0}^{1, p}(\Omega)$. Moreover, for almost every $x \in \Omega$, one has $|L u(x)| \leq e|h(x)|$.

Proof. For every $(x, z) \in \Omega \times \mathbb{R}$, we set $f(x, z)=h(x) e^{|z|}$. Obviously, the function $f: \Omega \times \mathbb{R} \rightarrow \mathbb{R}$ so defined satisfies the assumptions $\left(\mathrm{k}_{1}\right)$ and $\left(\mathrm{k}_{2}\right)$ of Theorem 2.3. Moreover, if we pick $r=B^{-1}$, then by (9), one has

$$
\left(\int_{\Omega}\left(\sup _{|z| \leq B r}|f(x, z)|\right)^{p} d x\right)^{1 / p}=e\|h\|_{L^{p}(\Omega)} \leq \frac{1}{B} .
$$

This implies that $\left(\mathrm{k}_{3}\right)$ of Theorem 2.3 holds. Hence, by that result, there exists $u \in W^{2, p}(\Omega) \cap W_{0}^{1, p}(\Omega)$ such that $L u(x)=h(x) e^{|u(x)|}$ almost everywhere in $\Omega, u(x)=0$ for all $x \in \partial \Omega$, and $|L u(x)| \leq e|h(x)|$ for almost every $x \in \Omega$.

Acknowledgements. The author wishes to thank Professors F. Chiarenza and F. Guglielmino for useful conversations.

\section{References}

[1] R. A. Adams, Sobolev Spaces, Academic Press, 1975.

[2] A. Alvino e G. Trombetti, Sulle migliori costanti di maggiorazione per una classe di equazioni ellitiche degeneri e non, Ricerche Mat. 30 (1981), 15-33.

[3] H. Amann, Nonlinear operators in ordered Banach spaces and some applications to nonlinear boundary value problems, in: Lecture Notes in Math. 543, Springer, 1976, 1-55.

[4] Yu. G. Borisovich, B. D. Gel'man, A. D. Myshkis and V. V. Obukhovskii, Multivalued mappings, J. Soviet Math. 24 (1984), 719-791.

[5] K. C. Chang, The obstacle problem and partial differential equations with discontinuous nonlinearities, Comm. Pure Appl. Math. 33 (1980), 117-146.

[6] - Variational methods for nondifferentiable functionals and their applications to partial differential equations, J. Math. Anal. Appl. 80 (1981), 102-129.

[7] D. Gilbarg and N. S. Trudinger, Elliptic Partial Differential Equations of Second Order, 2nd ed., Springer, 1983.

[8] C. J. Himmelberg, Measurable relations, Fund. Math. 87 (1975), 53-72.

[9] J. K. Kazdan and F. W. Warner, Remarks on some quasilinear elliptic equations, Comm. Pure Appl. Math. 28 (1975), 567-597.

[10] S. A. Marano, Existence theorems for a multivalued boundary value problem, Bull. Austral. Math. Soc. 45 (1992), 249-260. 
[11] J. Mawhin, J. R. Ward, Jr., and M. Willem, Variational methods and semilinear elliptic equations, Arch. Rational Mech. Anal. 95 (1986), 269-277.

[12] C. Miranda, Partial Differential Equations of Elliptic Type, 2nd revised ed., Springer, 1970.

[13] O. Naselli Ricceri and B. Ricceri, An existence theorem for inclusions of the type $\Psi(u)(t) \in F(t, \Phi(u)(t))$ and application to a multivalued boundary value problem, Appl. Anal. 38 (1990), 259-270.

[14] C. A. Stuart, Maximal and minimal solutions of elliptic differential equations with discontinuous nonlinearities, Math. Z. 163 (1978), 239-249.

[15] C. A. Stuart and J. F. Toland, A variational method for boundary value problems with discontinuous nonlinearities, J. London Math. Soc. 21 (1980), 319-328.

[16] G. Talenti, Elliptic equations and rearrangements, Ann. Scuola Norm. Sup. Pisa Cl. Sci. (4) 3 (1976), 697-718.

[17] M. Zuluaga, Existence of solutions for some elliptic problems with critical Sobolev exponents, Rev. Mat. Iberoamericana 5 (1989), 183-193.

DIPARTIMENTO DI MATEMATICA

UNIVERSITÀ DI CATANIA

VIALE A. DORIA 6

95125 CATANIA, ITALY

Reçu par la Rédaction le 5.4.1993 